\title{
Article \\ A Novel Cooperative Transmission Scheme in UAV-Assisted Wireless Sensor Networks
}

\author{
Yue Zang ${ }^{1}$, Yuyang Peng ${ }^{1, *}$, Sangdon Park ${ }^{2} \mathbb{D}$, Han Hai $^{3} \mathbb{D}$, Fawaz AL-Hazemi ${ }^{4}(\mathbb{D}$ \\ and Mohammad Meraj Mirza 5 iD
}

check for updates

Citation: Zang, Y.; Peng, Y.; Park, S.; Hai, H.; AL-Hazemi, F.; Mirza, M.M. A Novel Cooperative Transmission Scheme in UAV-Assisted Wireless Sensor Networks. Electronics 2022, 11, 600. https://doi.org/10.3390/ electronics 11040600

Academic Editors: Martin Reisslein and Christos J. Bouras

Received: 27 November 2021

Accepted: 3 February 2022

Published: 15 February 2022

Publisher's Note: MDPI stays neutral with regard to jurisdictional claims in published maps and institutional affiliations.

Copyright: (C) 2022 by the authors. Licensee MDPI, Basel, Switzerland. This article is an open access article distributed under the terms and conditions of the Creative Commons Attribution (CC BY) license (https:// creativecommons.org/licenses/by/ $4.0 /)$.
1 Faculty of Information Technology, Macau University of Science and Technology, Macau SAR 999078, China 1809853jii200109@student.must.edu.mo

2 Department of Electrical Engineering, Korea Advanced Institute of Science and Technology, Daejeon 34141, Korea; sangdon.park@kaist.ac.kr

3 College of Information Science and Technology, Donghua University, Shanghai 201620, China; hhai@dhu.edu.cn

4 Department of Computer and Network Engineering, University of Jeddah, Jeddah 21959, Saudi Arabia; fmalhazemi@uj.edu.sa

5 Department of Computer Science, College of Computers and Information Technology, Taif University, P.O. Box 11099, Taif 21944, Saudi Arabia; mmmirza@tu.edu.sa

* Correspondence: yypeng@must.edu.mo

\begin{abstract}
In wireless sensor networks (WSNs), the efficiency of data transmission within a limited time is critical, especially for sensors designed with small batteries. In this paper, we design a cooperative transmission scheme with an energy-charging function in a WSN where an unmanned aerial vehicle $(\mathrm{UAV})$ is considered for sensory data collection and energy charging. Specially, the sensor nodes are powered by the UAV for their data transmission. In the first phase, the UAV transmits the energy signal to the sensor nodes distributed on the ground. All the energy received by the sensor nodes is used to collect and transmit the sensory data to the UAV. In the second phase, local data transmissions are conducted among the collaborating sensor nodes in one cluster. In the third phase, the cooperative nodes send the collected sensory data to the UAV in the form of cooperative transmission. In the proposed scheme, we discovered that the size of the modulation constellation and the assigned time ratio of each phase were the key factors affecting the data transmission efficiency. In order to achieve the maximum data transmission, the optimal modulation constellation size and the optimal time ratio of each phase were found using the Lagrange multiplier method. Numerical results show that the proposed scheme with the optimal constellation size and the optimal time ratio can outperform the existing scheme in terms of the data transmission efficiency.
\end{abstract}

Keywords: WSNs; UAV; cooperative transmission; transmission efficiency

\section{Introduction}

With the rapid development of unmanned aerial vehicle (UAV)-related technology in the word, UAVs have developed wide applications and distinctive features. UAVs can be divided into military UAVs and civil UAVs according to their usage. Military UAVs are useful in target tracking and striking, while civil UAVs are useful in environmental monitoring, firefighting, farming, and good delivery. Currently, they are widely used in several areas that are difficult or inefficient for manual operation such as route inspection and remote sensing. For example, in the area of intelligent inspection, UAVs can detect blind spots that cannot be observed by humans, which can improve the efficiencies of inspection and the emergency rescue. In the area of agriculture, UAVs with spectrum analyzer and thermal infrared sensors can accurately calculate the planting area, which can improve the efficiency of farmland measuring. In addition, UAVs are also widely used in monitoring, wireless sensor networks (WSNs), and other fields [1]. 
Recently, the potential use of UAVs in WSNs has attracted great interest. Compared with traditional WSNs, WSNs supported by UAVs have several main advantages such as cost effectiveness and deployment flexibility. In WSNs, because sensor nodes are distributed in some areas with complex environments, the costs of collecting sensing data and maintaining wireless sensor nodes are very high. Therefore, placing nodes reasonably at the beginning can reduce these costs and extend the life of the sensors. Innovative node topology coverage is proposed in [2], which improves energy efficiency and coverage of node services. An effective node layout is important in any situation. However, due to the fact that some complex geographic locations and functional requirements, the placement of nodes is not always the most efficient method. Therefore, saving node's lifetime and improving the efficiency should be considered. For example, the authors in [3] propose a new clustering algorithm by applying adaptive cluster head selection method, which can improve the stability and save the energy of the network while maintaining the network connectivity. Moreover, in a clustered WSN, an energy-saving based multi-hop transmission strategy is proposed in [4] to minimize the power consumption of sensor nodes. The cluster head is used to transmit the received information to the maintenance center in order to take appropriate interventions. Compared with the direct transmission or the single relay transmission, the multi-hop transmission technology can greatly reduce power consumption. In the case of the traditional multi-hop transmission, the transmitter and receiver are fixed. However, in UAV-assisted WSNs, UAVs as mobile transceivers provide more possibilities for WSNs and need to communicate with more than one node in an area. In this way, the complex and time-consuming process of determining the optimal locations of UAVs hampers the development of UAV-assisted networks. To solve this problem, the authors of [5] proposed a multi-hop transmission method for UAV-assisted networks to improve energy efficiency. In another aspect, the combination of sensor nodes' cluster and UAVs can further save network resources. The authors of [6-9] proposed the optimal cluster head selection algorithm and protocol to maximize the lifetime of the WSN and improve the flexibility of the data collection using UAV. In [10], a UAV collecting a set of sensing data in a straight line was considered. By optimizing the transmitting power of the nodes and the flight speed of the UAV, the total flight time of the UAV from the starting point to the destination was minimized. In [11], when the sensing data collection and energy consumption of the UAV were limited, the hover position and duration of the UAV were controlled to save the energy of the sensor nodes. The trajectory of the UAV is another factor affecting energy efficiency. For example, the authors of [12] proposed a UAV data acquisition scheme by setting the optimal trajectory, which not only improves energy utilization, but also reduces data redundancy. In [13], interference can be managed by optimizing the transmission power of all communication nodes and planning the trajectory of the UAV to maximize the total throughput of target sensors. When a UAV collects data, network topology is constantly changing because the UAV is flying. It is essential to design an efficient data acquisition protocol for different dynamic situations to prolong the network lifetime [14-16]. In our work, we considered a UAV-assisted network and the design of an efficient transmission method. In addition, our work mainly focused on the physical layer technologies and, therefore, we assume that there was a perfect routing in the system model $[17,18]$. Unlike the methods proposed in most of the aforementioned works, the goal of our work was to transmit more data. In [19-21], we know that in WSNs, saving energy is no longer a single purpose, and time consumption must also be taken into consideration. Therefore, how to use the given energy in a limited time to transmit the most data is very meaningful [22,23]. In this paper, to solve the above problems, we jointly used a UAV with the cooperative multiple-input multiple-output (MIMO) scheme in WSNs. The objective was to transmit more data in a limited time, and the innovation was to discover the relationship between cooperative transmission and the transmission efficiency. In cooperative transmission, there are two parameters, including constellation size and time ratio, that can be investigated, and we jointly considered these two parameters to improve the transmission efficiency between the sensor nodes and the UAV. So 
far, there is no work considering these two parameters together in a UAV-based WSN. For a better understanding of the system's performance, we compared the proposed scheme with various benchmark schemes such as the optimized time ratio-optimized constellation SISO-based scheme, optimized time ratio-fixed constellation MIMO-based scheme, fixed time ratio-optimized constellation MIMO-based scheme, and fixed time ratio-fixed constellation MIMO-based scheme. The simulation results demonstrated that the proposed scheme had better performance in terms of data transmission when compared with other benchmark schemes.

The remainder of this paper is organized as follows. In Section 2, the system model is introduced. In Section 3, the optimal solution of the data transmission problem is given. Section 4 shows the experimental data and numerical results. The conclusions are given in Section 5 .

\section{System Model}

We considered a UAV-assisted WSN system as shown in Figure 1. In the system, the UAV is dynamic, and the sensor nodes are stationary. In other words, the sensor nodes are placed in advance and fixed in somewhere. Therefore, the distance between the sensor nodes was considered a fixed value. The UAV flying above the sensor nodes was equipped with one antenna for receiving sensing data, and each sensor node on the ground was embedded with one antenna for sensing data collection. The UAV collected data automatically and located the position of the sensor node by identifying the feedback signal of the sensor node. Before a UAV performs its operations, it first activates the nodes on the ground [24,25]. This process is conducted by transmitting an activation signal. The sensor nodes will be in idle status if they do not work for transmitting or receiving. In the idle status, the sensor nodes consume less energy than the case of working status. The duration depends on the workload. If the sensor nodes finish their tasks, they will change to the idle mode.

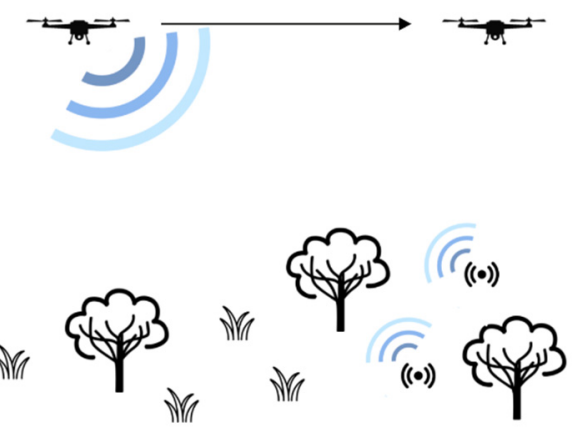

Figure 1. The structure of the UAV-assisted WSN.

In addition to receiving sensing data, the UAV can also be used as the energy provider. The UAV transmits the charging signal along with the activation signal to the sensors, and once the UAV receives the charging feedback signal, it will send the energy signal to the sensors. We assumed that the sensor nodes on the ground receive the energy signal from the UAV stably as shown in Figure 2a. The UAV charges the sensor nodes in turn. After charging the first node, it charges the second node. The energy received by the sensor nodes is assumed to use as the sensing data transmission. In order to transmit more data, it is better to use an energy-efficient method for data transmission. MIMO as a solution that can provide energy-efficient transmission in a fading channel environment. But usually, the sensor nodes are small and cannot be designed with multiple antennas to perform MIMO function. In order to utilize the MIMO properties, sensor nodes must collaborate with each other to form a virtual MIMO for cooperative transmission. In order to form a cooperative transmission, local data exchange is required before the long-distance transmission. In the local data exchange, each node uses a different time slot to broadcast its own information to other local nodes as shown in Figure 2(b1) and Figure 2(b2). This technique can be any time 
division technique such as time division multiple address (TDMA). When all nodes receive the information from others, cooperative sensor nodes encode the transmission sequence using the space time block code for cooperative transmission as shown in Figure 2c [17], and this step requires time synchronization. Usually, the synchronization can be achieved by means of beacon frames transmissions. A sensor node broadcasts its beacon frames in order to share its current schedule and status information with its cooperative nodes. In this way, all the sensor nodes can receive their neighbor's schedule and use this knowledge for data transmission. In this paper, we focused on physical layer technologies to investigate the data transmission. Therefore, such a MAC layer technology is out of the scope and will be omitted in our work. Compared with the single-input single-output (SISO) transmission scheme, cooperative transmission has an additional step, namely, the cooperative stage. In this paper, we applied the cooperative multiple-input single-output transmission (MISO) scheme as the cooperative stage in the UAV-assisted WSN. When sensor nodes work for a while, they need to be charged for working for the next round. Since such a scenario is usually applied in a remote area, we therefore assumed in such an area that there was no object between the UAV and ground. The energy consumption of the sensor node in the above three steps was formulated as follows:

$$
E_{E C}=E_{\text {local }}+E_{\text {long }}
$$

where $E_{\text {local }}$ represents the energy consumption of the local data exchange stage shown in Figure $2 \mathrm{~b}$, and $E_{\text {long }}$ is the energy consumption of the cooperative transmission shown in Figure 2c.

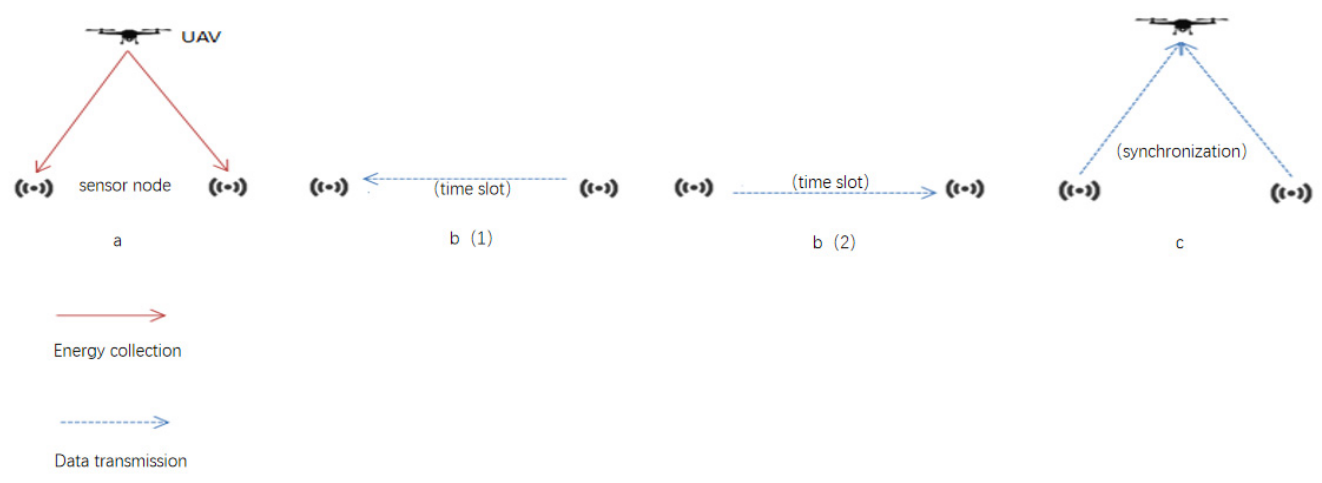

Figure 2. Three steps of energy charging and data transmission. (a) energy charging (b1,b2) local cooperation (c) cooperative transmission.

As we mentioned, our goal was to transmit more data within a certain time. Therefore, we considered the effect of the time duration. We assumed that $T$ was the total time composed of three stages, and $\alpha$ and $\beta$ represented the time ratio of the local and the cooperative transmission, respectively. Therefore, the remaining time $(1-n \alpha-\beta) \cdot T$ represents the UAV energy charging time. Consequently, in the step of energy charging, the energy received by all the sensor nodes is expressed as:

$$
E_{E H}=\eta \cdot P_{0}(1-n \alpha-\beta) \cdot T
$$

where $0<\eta \leq 1$ is the constant denoting the energy conversion efficiency, which is the degree of energy loss from the received signal to electric energy [26]; $n$ denotes the number of sensor nodes; $P_{0}$ is the power received by the sensor. In this work, we assumed that the sensor nodes could stably receive the energy from the UAV. We assumed that the energy of the UAV was infinite in the ideal case and the sensor nodes could always receive the maximum receiving power from the UAV. Each node needs to broadcast in different time slots; therefore, the number of time slots is $n$, and the time ratio of local cooperation is $n \alpha T$. In the real case, there can be many tasks depending on the different scenarios; however, the focus of our work was not on the multiple tasks. We were more interested in the 
optimization of the simple task scenario. Therefore, we provided a simple model on the UAV side and assumed that it had the simple task and enough energy. Note that this simple task model can be extended to the multiple tasks model by considering other tasks.

\subsection{Local Cooperation}

In the step of local cooperation, the channel was assumed to be a Rayleigh fading channel, and the gain between the transmitting node and receiving node was scalar [27,28]

The purpose of local cooperation is to prepare for cooperative transmission. Each sensor node must broadcast its own data to the other sensor nodes. In this step, we assumed that each sensor node had the same amount of data. In the local cooperation stage, the energy consumption of sensor nodes mainly contains two parts: circuit energy consumption and transmission energy consumption. The circuit energy consumption is the energy consumption of the circuit when it works for transmission, while the transmission energy consumption is the energy consumption for data transmission. Therefore, the energy of consumption local cooperation is expressed as [29]:

$$
E_{\text {local }}=n \cdot P_{\text {SISO }}^{d} \frac{L_{1}}{R_{\text {local }}}+n\left[P_{T}+(n-1) P_{R}\right] \frac{L_{1}}{R_{\text {local }}}
$$

where $P_{S I S O}^{d}$ denotes the power consumption of the node amplifier, $P_{T}$ and $P_{R}$ represent the power consumption of other circuits used in the transmitter and receiver, respectively, $L_{1}$ denotes the amount of data for each node, $R_{\text {local }}=b_{1} \cdot B$ denotes the transmission data rate with $b_{1}$ being the size of constellation in local cooperation and $B$ being the modulation bandwidth. Each sensor node transmits once; therefore, each sensor node receives $n-1$ times of the transmitted data. In Equation (3), $P_{S I S O}^{d}$ can be expressed as follows [17]:

$$
P_{S I S O}^{d}=\left(1+\alpha_{1}\right) \bar{E}_{\text {local }} R_{\text {local }} \frac{(4 \pi d)^{2}}{G_{t} G_{r} \lambda^{2}} M_{l} N_{f}
$$

where $M_{l}$ is the link margin compensating the hardware process variations and other additive background noise or interference; $N_{f}$ is the receiver noise level defined as $N_{f}=\left(N_{r} / N_{0}\right)$ with $N_{r}$ being the power spectral density (PSD) of the total effective noise at the receiver input and $N_{0}$ being the single-sided thermal noise PSD at room temperature; $G_{t}$ is the transmitter antenna gain; $G_{r}$ is the receiver antenna gain; $\alpha_{1}$ can be expressed as $\alpha_{1}=\left(\xi / \eta_{1}\right)-1$ with $\xi$ being the peak-to-average ratio (PAR) and $\eta_{1}$ being the drain efficiency of the radio frequency $(\mathrm{RF})$ power amplifier. The value of $\xi$ depends on the modulation scheme and constellation size. When multi-quadrature amplitude modulation (MQAM) is used in the local cooperation, $\xi$ is expressed as [30]:

$$
\xi=3\left(\frac{2^{\frac{b_{1}}{2}}-1}{2^{\frac{b_{1}}{2}}+1}\right) \text {. }
$$

In Equation (4), $\bar{E}_{\text {local }}$ is the energy per bit required for a given bit error ratio (BER) requirement, and it can be calculated according to the following relationship [17]:

$$
\bar{P}_{b_{1}} \approx \varepsilon_{H}\left\{\frac{4}{b_{1}}\left(1-\frac{1}{2^{\frac{b_{1}}{2}}}\right) Q\left(\sqrt{\frac{3 b_{1}}{M-1} \gamma_{b_{1}}}\right)\right\}
$$

under the constraint of $b_{1} \geq 2$ and:

$$
\bar{P}_{b_{1}} \approx \varepsilon_{H}\left\{Q \sqrt{2 \gamma_{b_{1}}}\right\}
$$


for $b_{1}=1$, where $\gamma_{b_{1}}=\left(\|H\|_{F}^{2} / M_{t}\right)\left(\bar{E}_{b_{1}} / N_{0}\right)$ and $M=2^{b_{1}}$. For simplicity, we used the error probability formula for MQAM with square constellations to approximate $\bar{P}_{b_{1}}$, and we can apply the Chernoff bound to obtain an upper limit shown as:

$$
\bar{E}_{\text {local }} \leq \frac{2}{3}\left(\frac{\bar{P}_{b_{1}}}{4}\right)^{-\frac{1}{M_{t}}} \frac{2^{b_{1}}-1}{b_{1}{ }^{\frac{1}{M_{t}}+1}} M_{t} N_{0}
$$

where $M_{t}$ denotes the number of antennas in the transmitter, and $N_{0}$ is the single-sided thermal noise. Therefore, the energy consumption of the local cooperation can be further expressed as:

$$
\begin{aligned}
E_{\text {local }}= & n \cdot\left(\frac{3}{3 \eta_{1}}\left(\frac{2^{\frac{b_{1}}{2}}-1}{2^{\frac{b_{1}}{2}}+1}\right) \cdot \frac{2}{3}\left(\frac{\bar{P}_{b_{1}}}{4}\right)^{-\frac{1}{M_{t}}}\right) \\
& \cdot\left(\frac{2^{b_{1}}-1}{b_{1}} M_{t} N_{0} R_{\text {local }} \frac{(4 \pi d)^{2}}{G_{t} G_{t} \lambda^{2}} M_{l} N_{f}\right) \frac{L_{1}}{R_{\text {local }}} \\
& +n\left[P_{T}+(n-1) P_{R}\right] \frac{L_{1}}{R_{\text {local }}} .
\end{aligned}
$$

\subsection{Cooperative Transmission}

Cooperative transmission is the final step of all the three steps, where cooperative sensor nodes work together to form a cooperative transmission to transmit the collected sensing data to the UAV. In this step, the Rayleigh fading channel with square-law path loss is assumed. Rayleigh fading is a statistical model for the effect of a propagation environment on a radio signal. The energy consumption of the cooperative transmission step is similar to the energy consumption of the local cooperation step which includes two parts, namely, circuit energy consumption and transmission energy consumption. Therefore, the energy consumption of the cooperative transmission is expressed as:

$$
E_{\text {long }}=P_{M I S O}^{D} \frac{L_{2}}{R_{\text {long }}}+n P_{T} \frac{L_{2}}{R_{\text {long }}}
$$

where $R_{\text {long }}$ is the data rate of cooperative transmission and determined by $B$ and the constellation size of cooperative transmission $b_{2}$ with the relation of $R_{\text {long }}=B \cdot b_{2} ; P_{M I S O}^{D}$ is the power consumption of power amplifier; $L_{2}$ is the amount of data transmitted in the cooperative transmission. Using a similar method to process Equation (10), it can be expressed as:

$$
\begin{aligned}
E_{\text {long }}= & \left(\frac{3}{3 \eta_{1}}\left(\frac{2^{\frac{b_{2}}{2}}-1}{2^{\frac{b_{2}}{2}}+1}\right) \cdot \frac{2}{3}\left(\frac{\bar{P}_{b_{2}}}{4}\right)^{-\frac{1}{M_{t}}}\right) \\
& \cdot\left(\frac{2^{b_{2}}-1}{\frac{1}{M_{t}}+1} M_{t} N_{0} R_{\text {long }} \frac{(4 \pi d)^{2}}{G_{t} G_{r} \lambda^{2}} M_{l} N_{f}\right) \cdot \frac{L_{2}}{R_{\text {long }}} \\
& +n P_{T} \frac{L_{2}}{R_{\text {long }}} .
\end{aligned}
$$

In the following, we obtained the relationship between the energy consumption and the transmission time of each stage by analyzing the system model. Then we could analyze the impact of each parameter on the amount of data transmission. In each parameter, we need to find the optimal value so that the sensor nodes can transmit the most data in the given time by using the proposed transmission scheme.

\section{Optimal Solution of the Data Transmission}

It was verified that in non-cooperative transmission schemes, the amount of transmitted data can be maximized by controlling the transmission power and the time ratio of energy charging and data transmitting [19]. In the proposed scheme, instead of controlling the transmitting power, we chose the modulation constellation size as the controlling factor. In addition, we discovered the best time ratio of each of the three steps. By choosing these two factors together, we could obtain the optimal amount of data transmission. In the process of solving the optimization problem, we made the following assumptions. We 
assumed that the distance between two activated sensor nodes was one meter. When the UAV collected data, it was located directly above the two sensor nodes. The local transmission did not contain data compression.

Next, we analyzed the impact of changing the constellation size. From Equations (9) and (11), we know that the size of the constellation affects the energy consumption. From the aforementioned analysis, we know that the constellation size also affects the transmission rate. Obviously, it is impossible to increase the transmission rate while decreasing the energy consumption. We first consider the effect of changing the rate on the total amount of transmitted data. We first consider the influence of constellation size on the total data transmission. For $b_{1}$, we refer to the value of 12 [17]. For $b_{2}$, we know from Equation (11) that it is related to the time ratio of local cooperation. We can get the specific relationship between the transmitted data in the following two stages as shown as:

$$
\beta T \cdot B \cdot b_{2}=2 \alpha T \cdot B \cdot b_{1}
$$

where the right side of the formula represents the total amount of data transmitted by the sensor nodes in the local transmission step, and the left side of the formula represents the amount of data that sensor nodes cooperatively transmit to the UAV.

As mentioned in system model, all the received energy was used for data transmission including the local cooperation and the cooperative transmission. This relation can be expressed as follows:

$$
\begin{aligned}
\eta \cdot P_{0}(1-2 \alpha-\beta) T= & \left\{n \cdot P_{S I S O}^{d}+n\left[P_{T}+(n-1) P_{R}\right]\right\} \alpha T \\
& +\left(P_{M I S O}^{D}+n P_{T}\right) \cdot \beta T .
\end{aligned}
$$

Therefore, the optimization problem can be formulated as follows:

$$
\begin{aligned}
\max _{b_{2}, \beta}=\beta T & \cdot B \cdot b_{2}=2 \alpha T \cdot B \cdot b_{1} \\
\text { s.t } \quad \eta P_{0}(1-2 \alpha-\beta)= & \left\{n \cdot P_{S I S O}^{d}+n\left[P_{T}+(n-1) P_{R}\right]\right\} \alpha \\
+ & \left(P_{M I S O}^{D}+n P_{T}\right) \beta .
\end{aligned}
$$

We use the Lagrange method to determine the maximum amount of data. The first step was to construct the Lagrange function shown as follows:

$$
\begin{aligned}
L(\alpha, \beta, \phi) & =b_{2} B \cdot \beta+\phi\left\{\left(\eta \cdot P_{0}(1-2 \alpha-\beta)\right)\right. \\
& -\left\{n \cdot P_{S I S O}^{d}-n\left[P_{T}+(n-1) P_{R}\right]\right\} \frac{b_{2}}{2 b_{1}} \beta \\
& \left.-\left(P_{M I S O}^{D}+n P_{T}\right) \cdot \beta\right\}
\end{aligned}
$$

where $\phi$ is Lagrange multiplier. The maximum value must be the tangent point that can be tangent to the constraint condition curve among the multiple contour curves of the objective function. We took the partial derivative of Equation (15) with respect to $\phi$ and set it to 0 , and following equations could be obtained:

$$
\begin{aligned}
\eta \cdot P_{0}(1-2 \alpha-\beta)= & \left\{n \cdot P_{S I S O}^{d}+n\left[P_{T}+(n-1) P_{R}\right]\right\} \alpha \\
& +\left(P_{M I S O}^{D}+n P_{T}\right) \cdot \beta .
\end{aligned}
$$

We rewrote Equation (16) and the following relationship could be obtained as shown:

$$
\beta=\frac{\eta \cdot P_{0}}{\left\{n P_{S I S O}^{d}+n\left[P_{T}+(n-1) P_{R}\right]\right\} \frac{b_{2}}{2 b_{1}}+\left(P_{M I S O}^{D}+n P_{T}\right)+\left(\frac{b_{2}+b_{1}}{b_{1}}\right) \eta P} .
$$


Next, we took the partial derivative of $L\left(b_{2}, \beta, \phi\right)$ with respect to $\beta$ and $b_{2}$, and the following equations could be obtained:

$$
\begin{aligned}
\frac{\partial L(\alpha, \beta, \phi)}{\partial \beta} & =b_{2} B+\phi\left(-\eta \cdot P_{0}\left(\frac{b_{2}+b_{1}}{b_{1}}\right)\right. \\
& -\left\{n \cdot P_{S I S O}^{d}+n\left[P_{T}+(n-1) P_{R}\right]\right\} \frac{b_{2}}{2 b_{1}} \\
& \left.-\left(P_{M I S O}^{D}+n P_{T}\right)\right) \\
\frac{\partial L(\alpha, \beta, \phi)}{\partial b_{2}} & =\beta B+\phi\left(-\eta \cdot P_{0}\left(\frac{\beta}{b_{1}}\right)\right. \\
& -\left\{n \cdot P_{S I S O}^{d}+n\left[P_{T}+(n-1) P_{R}\right]\right\} \frac{\beta}{2 b_{1}} \\
& \left.-\left(\frac{\partial P_{M I S O}^{D}}{\partial b_{2}} \cdot \beta\right)\right) .
\end{aligned}
$$

Solving Equations (18) and (19) and combining with Equation (16), Equation (20) could be determined as:

$$
\begin{aligned}
& \eta \cdot P_{0}\left(\frac{b_{2}}{b_{1}}\right)+\left\{n \cdot P_{S I S O}^{d}+n\left[P_{T}+(n-1) P_{R}\right]\right\} \frac{b_{2}}{2 b_{1}}+\left(\frac{\partial P_{M I S O}^{D}}{\partial b_{2}}\right) \cdot b_{2}= \\
& \eta \cdot P_{0}\left(\frac{b_{2}+b_{1}}{b_{1}}\right)+\left\{n \cdot P_{S I S O}^{d}+n\left[P_{T}+(n-1) P_{R}\right]\right\} \frac{b_{2}}{2 b_{1}}+\left(P_{M I S O}^{D}+n P_{T}\right) .
\end{aligned}
$$

After simplification of Equation (20), we obtained:

$$
\eta P_{0}-\left(\frac{\partial P_{M I S O}^{D}}{\partial b_{2}}\right) \cdot b_{2}+\left(P_{M I S O}^{D}+n P_{T}\right)=0 .
$$

Since the final solution of the equation was a transcendental equation, we used a numerical method to solve it.

In order to verify the performance of our results, we compared our results with different situations. In our method, we optimize the data transmission in a limited time by controlling two important factors, namely, the time ratio and the size of the modulation constellation. For the traditional transmission scheme, SISO-based transmission was used as the transmission scheme in the UAV-assisted WSNs. First, in the proposed scheme, we compared the one optimized factor's situation with a two fixed factors' situation. Then, we compared the two optimized factors' situation with one optimized factor's situation. Following that, the comparison of two optimized factors' situation with two fixed factors' situation was given. Finally, the traditional scheme with a two optimized factors' situation was compared with the proposed scheme with two optimized factors' situation.

\section{Results}

In this section, we numerically evaluated the performance of the proposed system. From our system model, we compared single optimization and full optimization in different cases. The related circuit and system parameters are given in Table 1 . Table 2 gives the result of the best time ratio versus different transmission distances. From Table 2, we can see that the time ratio changed along with the changes in the transmission distance.

Table 1. System parameters.

$\begin{array}{cc}\eta_{1}=0.35 & G_{t} G_{r}=5 \mathrm{dBi} \\ \bar{P}_{b}=10^{-3} & B=10 \mathrm{KHz} \\ N_{f}=10 \mathrm{~dB} & M_{l}=40 \mathrm{~dB} \\ P_{T}=150 \mathrm{~mW} & P_{R}=100 \mathrm{~mW} \\ \lambda=0.12 \mathrm{~m} & N_{0}=-171 \mathrm{dBm} / \mathrm{Hz} \\ n=2 & P_{0}=1.5 \mathrm{~W}\end{array}$

Figure 3 shows optimal constellation sizes versus different transmission distance values. We can see that in each transmission distance, an optimal constellation size can be found to optimize the data transmission. In Figure 4, we compare the proposed scheme with the optimized constellation-fixed ratio to the one with the fixed constellation-fixed 
ratio. In the experiment, the fixed constellation size for cooperative transmission was set to 14 , and the fixed time ratio for charging was set to 0.2 as an example. It can be seen from Figure 4, that after optimizing the constellation size, the amount of data transmission was greatly improved. In order to show the effectiveness of the time ratio optimization, we also compared the proposed scheme with optimized ratio-fixed constellation to the one with the fixed ratio-fixed constellation. The results in Figure 5 show that the proposed scheme can transmit more data after optimizing the time ratio.

Table 2. Constellation sizes at different distances.

\begin{tabular}{cccccc}
\hline Distance D $(\mathrm{m})$ & 10 & 20 & 30 & 40 & 50 \\
\hline Best ratio $\beta$ & 0.453 & 0.450 & 0.461 & 0.484 & 0.515 \\
\hline Distance D $(\mathrm{m})$ & 60 & 70 & 80 & 90 & 100 \\
\hline Best ratio $\beta$ & 0.497 & 0.539 & 0.525 & 0.510 & 0.494 \\
\hline
\end{tabular}

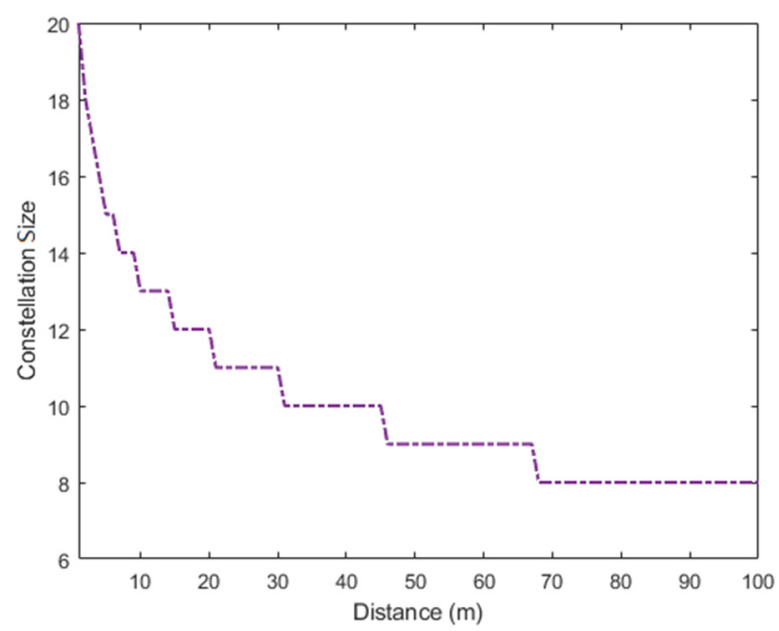

Figure 3. Optimal constellations at different distances.

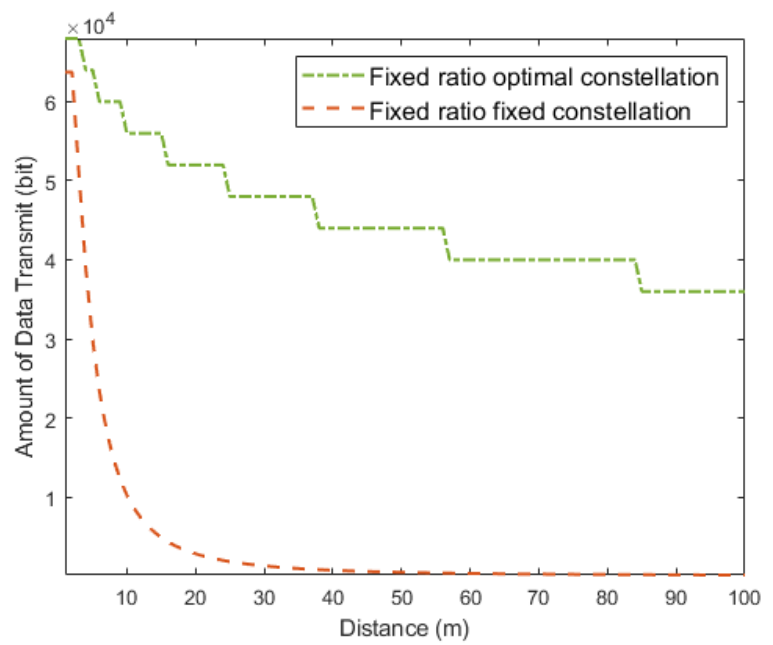

Figure 4. Fixed constellation versus optimized constellation size at a fixed time ratio.

The experimental results in Figures 4 and 5 show that optimizing the constellation size and the time ratio can effectively increase the amount of transmitted data within a given time. In order to prove that combining these two methods together can further improve the efficiency of data transmission, we sequentially compared the proposed scheme with two optimized factors to the one with single optimized factor. The result in Figure 6 shows that the proposed scheme with the two optimized factor has advantages over 
the one with the single optimized factor in terms of the data transmission amount. In addition to these comparisons, we also compared the proposed scheme with the common cooperative transmission scheme, namely, UAV-based cooperative transmission with a fixed constellation size and fixed time ratio. We set the transmission data as the common aim of the system to make the comparison fair. We found that the system's performance, in terms of data transmission, can further be improved by optimizing both constellation size and time ratio. Figure 7 shows the comparative result between these two schemes. It shows that the cooperative transmission scheme with both optimized factors can outperform the cooperative transmission scheme without optimized factors.

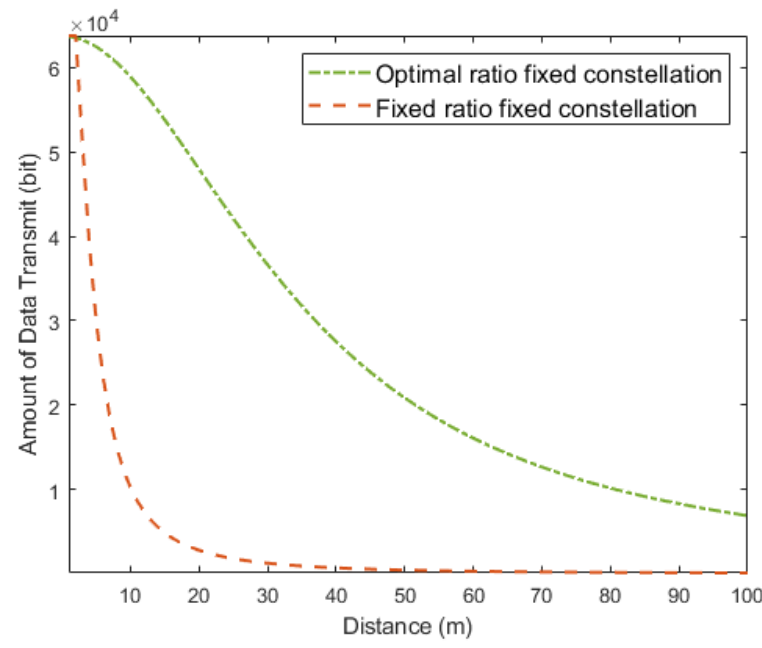

Figure 5. Fixed time ratio versus optimized time ratio at a fixed constellation.

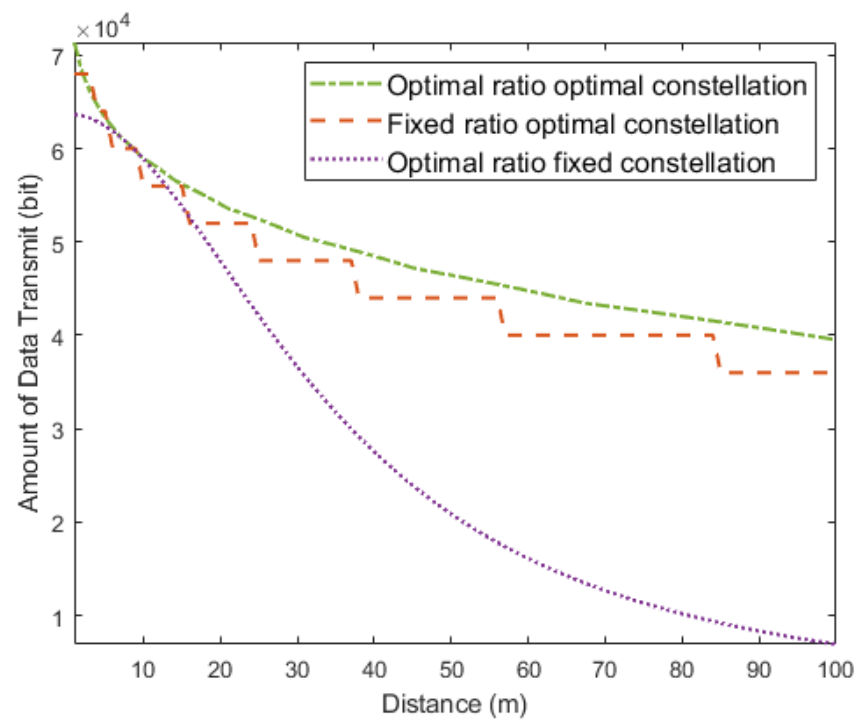

Figure 6. Optimized time ratio-optimized constellation size versus single optimized factor.

In some efficient transmission schemes, SISO with optimized time ratio is used as the transmission scheme [19]. In order to show the performance of the proposed scheme, we also compare it with the traditional scheme, namely, SISO-based UAV-assisted WSN $[19,22,30]$. The result in Figure 8 shows that there was a threshold and within the threshold, the SISO-based optimal scheme can beat the MISO-based optimal scheme, whereas out of the threshold the MISO-based optimal scheme can beat the SISO-based optimal scheme. 


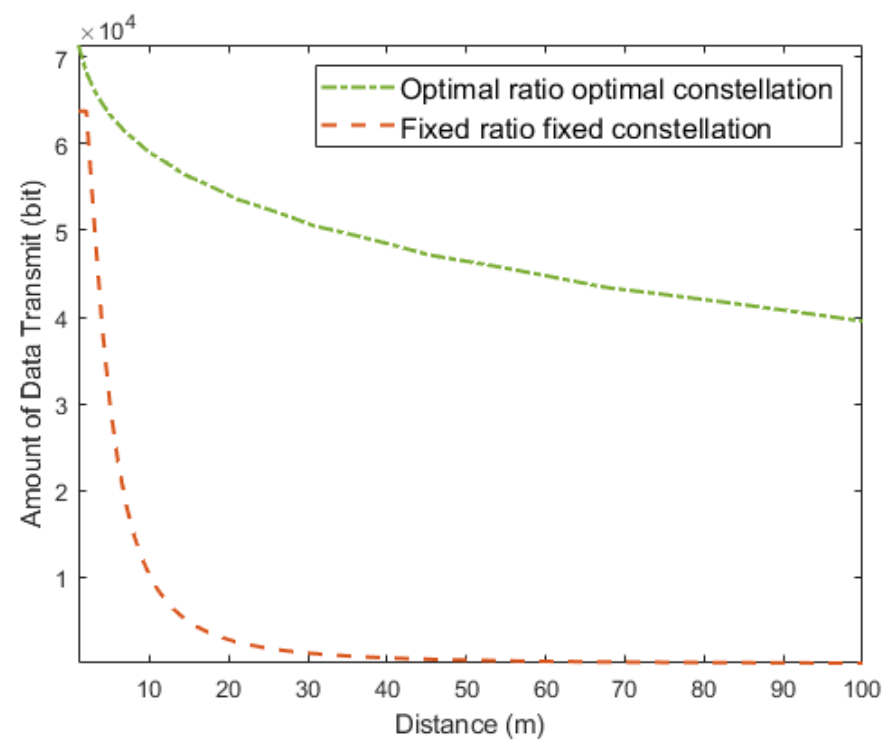

Figure 7. Optimized time ratio-optimized constellation size versus fixed time ration fixed constellation size.

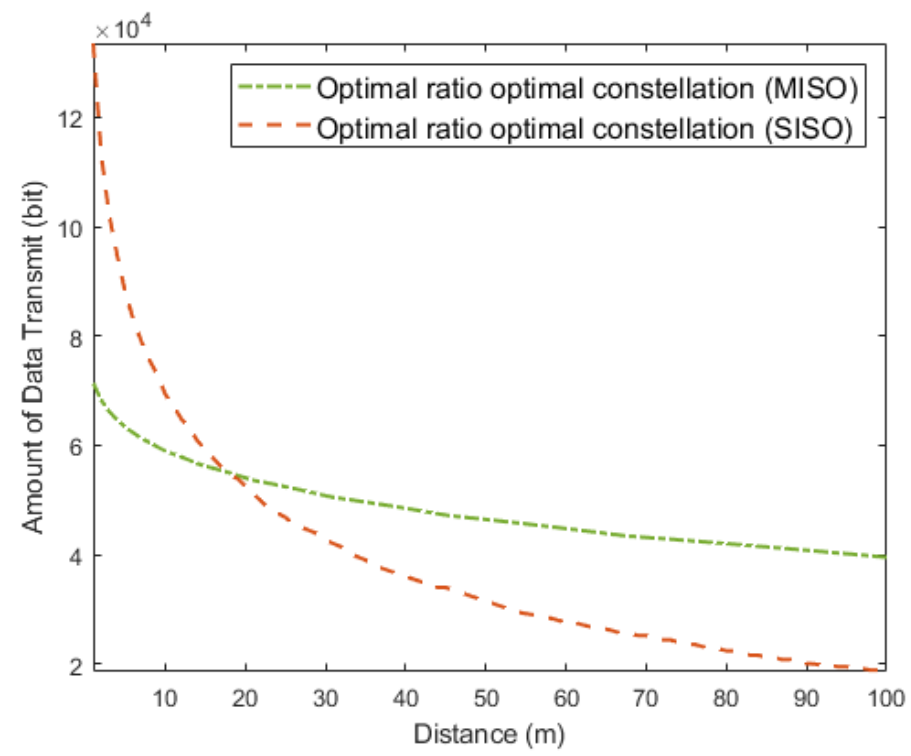

Figure 8. Comparison of MISO and SISO with the optimized constellation size optimized-time ratio.

\section{Conclusions}

In this article, we considered a cooperative transmission scheme for a UAV-assisted WSN with an energy charging function. In the analysis of the system model, we found that the constellation size directly affected the transmission rate and energy consumption. Therefore, when we then chose the appropriate constellation size at different transmission distances, the energy utilization and data transmission could be improved. In addition, in a certain period, a good time ratio of charging and data transmission can improve data transmission efficiency. Through these two aspects, we used the Lagrange multiplier method to obtain the optimal constellation size and time ratio, which maximized the data transmission. The results showed that the scheme with the optimized time ratio and optimized constellation size performed better than the one with a single optimized factor or non-optimized factor transmission schemes. We also compared the proposed scheme with optimized factors to the SISO-based scheme with optimized factors. The results showed that in short transmission distances, the SISO-based scheme performed better. But as the distance increased, the proposed scheme was more transmission efficient. 


\begin{abstract}
Author Contributions: Conceptualization, Y.Z. and Y.P.; methodology, Y.Z.; validation, Y.Z.; formal analysis, Y.Z.; investigation, Y.Z.; writing-original draft preparation, Y.Z. and Y.P.; writing-review and editing, S.P. and H.H.; visualization, Y.Z.; supervision, Y.P.; project administration, Y.P. and S.P.; funding acquisition, Y.P., F.A.-H., M.M.M. and S.P. All authors have read and agreed to the published version of the manuscript.
\end{abstract}

Funding: This work was supported in part by The Science and Technology Development Fund, Macau SAR (0108/2020/A3), in part by The Science and Technology Development Fund, Macau SAR $(0005 / 2021 /$ ITP), and in part by Taif University Researchers Supporting Project number (TURSP2020/329), Taif University, Taif, Saudi Arabia.

Acknowledgments: The authors are grateful for the support of Taif University Researchers Supporting Project number (TURSP-2020/329) Taif University, Taif, Saudi Arabia.

Conflicts of Interest: The authors declare that there are no conflicts of interest regarding the publication of this paper.

\title{
References
}

1. Keyworth, S.; Wolfe, S. UAVs for land use applications: UAVs in the civilian airspace institution of engineering and technology. In IET Seminar on UAVs in the Civilian Airspace; IET: London, UK, 2013; pp. 1-13.

2. Tharakan, L.; Dhanasekaran, R. Energy and coverage efficiency using straight line node deployment with data compression in Wireless sensor network. In Proceedings of the 2016 International Conference on Advanced Communication Control and Computing Technologies (ICACCCT), Ramanathapuram, India, 25-27 May 2016.

3. Ebadi, S.; Ghasembaglou, M.; Navin, A.H.; Mirnia, M.K. Energy Balancing in Wireless Sensor Networks with Selecting Two Cluster-Heads in Hierarchical Clustering. In Proceedings of the International Conference on Computational Intelligence and Communication Networks, Bhopal, India, 26-28 November 2010.

4. Herhold, P.; Zimmermann, E.; Fettweis, G. Cooperative multi-hop transmission in wireless networks. Comput. Netw. 2005, 49, 299-324. [CrossRef]

5. Abderrahim, M.; Hakim, H.; Boujemaa, H.; al Hamad, R. Multihop Transmission Strategy to Improve Energy Efficiency in WSNs. In Proceedings of the 32nd International Conference on Advanced Information Networking and Applications Workshops (WAINA), Krakow, Poland, 16-18 May 2018; pp. 136-140.

6. Haider, S.K.; Jamshed, M.A.; Jiang, A.; Pervaiz, H.; Ni, Q. UAV-assisted Cluster-head Selection Mechanism for Wireless Sensor Network Applications. In Proceedings of the UK/China Emerging Technologies (UCET), Glasgow, UK, 21-22 August 2019; pp. 1-2.

7. Tazibt, C.Y.; Bekhti, M.; Djamah, T.; Achir, N.; Boussetta, K. Wireless sensor network clustering for UAV-based data gathering. In Proceedings of the Wireless Days, Porto, Portugal, 29-31 March 2017; pp. 245-247.

8. Wang, G.; Lee, B.; Ahn, J.Y. UAV-Assisted Cluster Head Election for a UAV-Based Wireless Sensor Network. In Proceedings of the IEEE 6th International Conference on Future Internet of Things and Cloud (FiCloud), Barcelona, Spain, 6-8 August 2018; pp. 267-274.

9. Kashuba, S.V.; Novikov, V.I.; Lysenko, O.I.; Alekseeva, I.V. Optimization of UAV path for wireless sensor network data gathering. In Proceedings of the IEEE International Conference Actual Problems of Unmanned Aerial Vehicles Developments (APUAVD), Kiev, Ukraine, 13-15 October 2015; pp. 280-283.

10. Gong, J.; Chang, T.; Shen, C.; Chen, X. Flight Time Minimization of UAV for Data Collection Over Wireless Sensor Networks. IEEE J. Sel. Areas Commun. 2018, 36, 1942-1954. [CrossRef]

11. Baek, J.; Han, S.I.; Han, Y. Optimal UAV Route in Wireless Charging Sensor Networks. IEEE Internet Things J. 2019, 7, 1327-1335. [CrossRef]

12. Liu, X.; Liu, Y.; Zhang, N.; Wu, W.; Liu, A. Optimizing Trajectory of Unmanned Aerial Vehicles for Efficient Data Acquisition: A Matrix Completion Approach. IEEE Internet Things J. 2019, 6, 1829-1840. [CrossRef]

13. Zhang, S.; Shi, S.; Gu, S.; Gu, X. Power Control and Trajectory Planning Based Interference Management for UAV-Assisted Wireless Sensor Networks. IEEE Access 2019, 8, 3453-3464. [CrossRef]

14. Ma, X.; Kacimi, R.; Dhaou, R. Fairness-Aware UAV-Assisted Data Collection in Mobile Wireless Sensor Networks. In Proceedings of the International Wireless Communications and Mobile Computing Conference (IWCMC), Paphos, Cyprus, 5-9 September 2016; pp. 995-1001.

15. Kenyeres, M.; Kenyeres, J. Average Consensus over Mobile Wireless Sensor Networks: Weight Matrix Guaranteeing Convergence without Reconfiguration of Edge Weights. Sensors 2020, 20, 3677. [CrossRef] [PubMed]

16. Ma, X.; Liu, T.; Liu, S.; Kacimi, R.; Dhaou, R. Priority-Based Data Collection for UAV-Aided Mobile Sensor Network. Sensors 2020, 20, 3034. [CrossRef] [PubMed]

17. Cui, S.; Goldsmith, A.; Bahai, A. Energy-Efficiency of MIMO and Cooperative MIMO Techniques in Sensor Networks. IEEE J. Sel. Areas Commun. 2004, 22, 1089-1098. [CrossRef] 
18. Grira, L.; Bouallegue, R. Energy Efficiency of Cooperative MIMO in Wireless Sensor Networks over Rayleigh Fading Channel. In Proceedings of the IEEE 31st International Conference on Advanced Information Networking and Applications (AINA), Taipei, Taiwan, 27-29 March 2017; pp. 107-111.

19. Liu, Y.; Dai, H.-N.; Wang, H.; Imran, M.; Wang, X.; Shoaib, M. UAV-enabled data acquisition scheme with directional wireless energy transfer for Internet of Things. Comput. Commun. 2020, 155, 184-196. [CrossRef]

20. Peng, Y.; Al-Hazemi, F.; Boutaba, R.; Tong, F.; Hwang, I.-S.; Youn, C.-H. Enhancing Energy Efficiency via Cooperative MIMO in Wireless Sensor Networks: State of the Art and Future Research Directions. IEEE Commun. Mag. 2017, 55, 47-53. [CrossRef]

21. Peng, Y.; Al-Hazemi, F.; Kim, H.; Youn, C.-H. Design and Optimization for Energy-Efficient Cooperative MIMO Transmission in Ad Hoc Networks. IEEE Trans. Veh. Technol. 2016, 66, 1. [CrossRef]

22. Peng, Y.; Youn, C. Lifetime and energy optimization in multi-hop wireless sensor networks with spatial modulation based cooperative MIMO. IEEJ Trans. Electr. Electron. Eng. 2015, 10, 731-732. [CrossRef]

23. Peng, Y.; Li, J.; Park, S.; Zhu, K.; Hassan, M.; Alsanad, A. Energy-efficient cooperative transmission for intelligent transpor-tation systems. Future Gener. Comput. Syst. 2019, 94, 634-640. [CrossRef]

24. Kouzayha, N.; ElSawy, H.; Dawy, Z.; Andrews, J.G. Analysis of an ID-Based RF Wake-Up Solution for IoT over Cellular Networks. In Proceedings of the IEEE GLOBECOM Workshops, Singapore, 4-8 December 2017; pp. 1-6.

25. Chen, J.; Dai, Z.; Chen, Z. Development of Radio-Frequency Sensor Wake-Up with Unmanned Aerial Vehicles as an Aerial Gateway. Sensors 2019, 19, 1047. [CrossRef] [PubMed]

26. Wang, F.; Xu, J.; Wang, X.; Cui, S. Joint Offloading and Computing Optimization in Wireless Powered Mobile-Edge Computing Systems. IEEE Trans. Wirel. Commun. 2018, 17, 1784-1797. [CrossRef]

27. Paulraj, A.; Nabar, R.; Gore, D. Introduction to Space-Time Wireless Communications; Cambridge University Press: Cambridge, UK, 2003

28. Huang, Z.; Peng, Y.; Li, J.; Tong, F.; Zhu, K.; Peng, L. Secrecy Enhancing of SSK Systems for IoT Applications in Smart Cities. IEEE Internet Things J. 2021, 8, 6385-6392. [CrossRef]

29. Gao, Q.; Zuo, Y.; Zhang, J.; Peng, X.-H. Improving Energy Efficiency in a Wireless Sensor Network by Combining Cooperative MIMO With Data Aggregation. IEEE Trans. Veh. Technol. 2010, 59, 3956-3965. [CrossRef]

30. Cui, S.; Goldsmith, A.J.; Bahai, A. Energy-constrained modulation optimization. IEEE Trans. Wirel. Commun. 2005, 4, 2349-2360. 